\title{
Ex Vivo Optical Coherence Tomography Imaging of Collector Channels with a Scanning Endoscopic Probe
}

\author{
Jian Ren, ${ }^{1}$ Henrick K. Gille, ${ }^{2}$ Jigang $W u,{ }^{1}$ and Changbuei Yang ${ }^{1,3}$
}

Purpose. To achieve high-fidelity optical coherence tomography (OCT) imaging of ex vivo collector channels (CCs) exiting Schlemm's canal (SC) using a paired-angle rotating scanning endoscopic probe.

Methods. An endoscopic probe was developed to guide an OCT laser beam onto human cadaver eye tissue samples to detect CCs. The prototype probe consisted of two gradientindex (GRIN) lenses that were housed in two stainless steel needles, respectively. The probe scanned the laser beam across a fan shape area by rotating the two GRIN lenses. The authors built a swept source OCT system to provide the depth scans. Human cadaver eye tissue was prepared for imaging. OCT images were acquired while the wall of SC was scanned. After successfully locating the opening of a CC on the SC wall from the OCT images, the authors applied scanning electron microscopy (SEM) to image the sample for comparison.

Results. The prototype probe focused the laser beam to a working distance of approximately $1.4 \mathrm{~mm}$ (in air), with spot sizes ranging from 12 to $14 \mu \mathrm{m}$. The fan shape scan area had a radius of $3 \mathrm{~mm}$ and an arc angle of approximately $40^{\circ}$. Acquired OCT images clearly showed a CC opening on the wall of SC with the channel going into the sclera, from which quantitative measurements were made. Results from OCT and SEM show good agreement with each other.

Conclusions. The resolving power of the scanning endoscopic probe is sufficient to locate CCs and to observe their shape. (Invest Ophthalmol Vis Sci. 2011;52:3921-3925) DOI:10.1167/ iovs.10-6744

$\mathbf{M}$ ost open angle glaucomas result from the retention of aqueous humor. This is caused primarily by abnormalities in the trabecular meshwork (TM) that increase the resistance of aqueous humor outflow into Schlemm's canal (SC), thereby reducing physiological outflow through the collector channels (CCs) and the episcleral veins. ${ }^{1}$ Recently, stents have been developed to bypass the fluid resisting TM. For example, as shown in Figure 1a, the iStent (Glaukos Corporation, Laguna Hills, CA) is a micro bypass stent that is surgically implanted into the TM, effectively bypassing the obstructed meshwork,

From the Departments of ${ }^{1}$ Electrical Engineering and ${ }^{3}$ Bioengineering, California Institute of Technology, Pasadena, California; and ${ }^{2}$ Product Development, Glaukos Corporation, Laguna Hills, California.

Presented in part at the annual meeting of the Association for Research in Vision and Ophthalmology, Fort Lauderdale, Florida, May 2010.

Supported by National Science Foundation Biomimetic MicroElectronic Systems Engineering Research Center Grant EEC-0310723.

Submitted for publication October 18, 2010; revised January 5, 2011; accepted January 5, 2011

Disclosure: J. Ren, None; H.K. Gille, Glaukos Corporation (E); J. Wu, None; C. Yang, None

Corresponding author: Jian Ren, Department of Electrical Engineering, California Institute of Technology, $1200 \mathrm{E}$. California Boulevard, MC 136-93, Pasadena, CA 91125; jren@caltech.edu. thereby reestablishing physiological outflow of aqueous humor into SC. ${ }^{2,3}$

The implantation process involves introducing the stent into the anterior chamber through a corneal incision. It is advanced across the iris until it can be implanted in the TM. Recent research has shown that the SC cross-sectional area is wider in the nasal inferior quadrant of the anterior chamber, suggesting a greater prevalence of CCs draining that segment of SC. ${ }^{4}$ It is hypothesized that implanting a stent in closer proximity to these active CCs may increase fluid outflow. To test this hypothesis, it was first necessary to determine the location of the CCs.

Both high-resolution and depth-resolving capability have made optical coherence tomography (OCT) an important ophthalmic diagnostic tool. ${ }^{5,6}$ Given that scleral tissue and the TM are not transparent to visible light, OCT may be useful for visualizing and locating the CCs. Commercial spectral domain (SD)-OCT systems with a light source centered at $870 \mathrm{~nm}$ have been used to image through the sclera from the exterior surface of the eye (Kagemann L, et al. IOVS 2009;50:ARVO E-Abstract 813). ${ }^{4}$ However, there are two major problems with this method. First, the CCs were not clearly imaged. Both SC and its junctions with CCs (SC/CC) are not easily identified and located from those images. This can be attributed to the fact that the OCT beam coming from outside has been largely scattered by the scleral tissue, especially the blood in superficial vessels, before reaching SC. As a result, the image contrast of the structures inside scleral tissue, such as the SC/CC junctions, has been severely impaired. The shadowing effect by superficial blood vessels obscuring many regions of interest is one example (Kagemann L, et al. IOVS 2009;50:ARVO E-Abstract 813). ${ }^{4}$ Images of low contrast preclude the ability of surgeons to select the optimal implantation location of the stents during the operation. In addition, a number of axial scans (A scans) were averaged to improve the contrast (Kagemann L, et al. IOVS 2009;50:ARVO E-Abstract 813). ${ }^{4}$ This resulted in a scan time of 4.5 seconds, which makes real-time imaging impossible.

Second, for current commercial OCT systems, immobilization of the patient's head is required to provide stable images. For instance, in the previous studies (Kagemann L, et al. IOVS 2009;50:ARVO E-Abstract 813), ${ }^{4}$ a bite bar was used to reduce eye movement during the 4.5-second scan time, and visual inspection for eye movements between images was performed to subjectively select valid images. This limited the OCT examination to being carried out either before or after an operation. Because of the complex episcleral vein structures in the sclera, it is not easy to trace them back to SC during an operation even if they could have been located in a preacquired OCT image slice. Therefore, it is much more desirable to have a real-time imaging method that can view the surgical field from the inside of the anterior chamber during these surgeries.

OCT endoscopes offer a potential solution to these problems. They can be placed deep into tissues and collect reflected optical signals from the desired depth, providing im- 


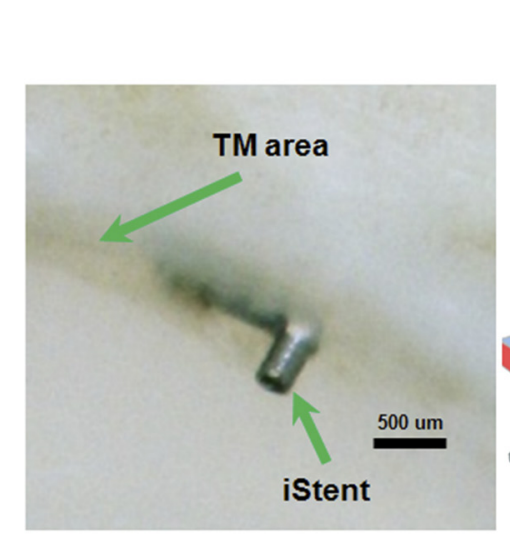

(a)

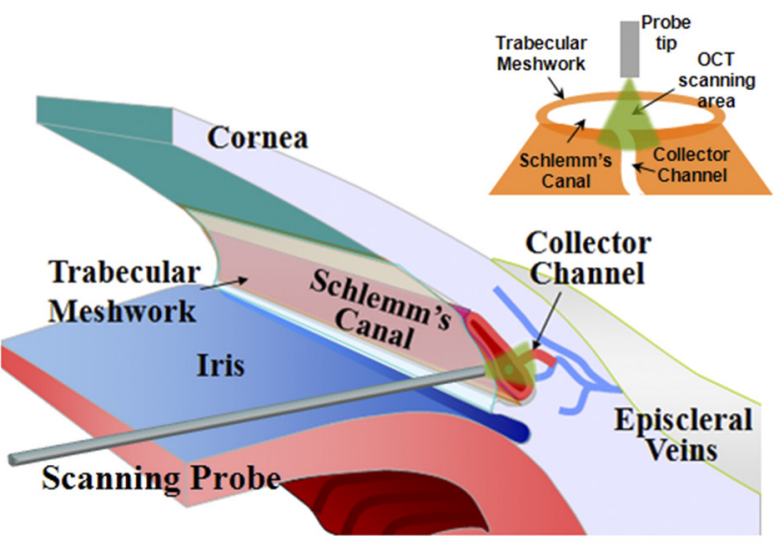

(b)
Figure 1. Glaucoma bypass stent implantation and its imaging guidance by an endoscopic OCT probe. (a) The iStent bypassing the TM. (b) Surgical configuration of the OCT imaging procedure using an endoscopic probe. Inset: relative positions of the physiological structures and the probe. Modified from a slide presented at the International Club for Biomaterials and Regenerative Medicine in Ophthalmology (ICBRO), April 2007. ages of much higher quality by overcoming signal attenuation from intervening tissue such as the sclera or TM. Furthermore, because of their small size, hand-held endoscopic probes can be used intraoperatively and are capable of providing real-time visualization and guidance for the specific structures of interest. ${ }^{7}$ Therefore, we propose an OCT endoscopic probe to determine the locations of CCs for the bypass stent implantation, as illustrated in Figure $1 \mathrm{~b}$. The proposed probe passes through the corneal incision for implantation and is advanced across the iris until it is in apposition to the TM, approximately 0.5 to $1 \mathrm{~mm}$ away. Then the probe starts scanning the forward cone in front of its tip in a fan-shape fashion. The OCT beam penetrates the TM, imaging the cross-section of the SC and disclosing the structures inside, such as CCs. The relative geometry between tissue structures and this probe is shown in the Figure $1 \mathrm{~b}$ inset. The goals of this research was to develop such an endoscopic OCT probe and to determine whether it is capable of locating the CCs exiting the SC and suitable for intraoperative ophthalmic applications.

\section{Methods}

\section{Scanning Endoscopic Probe}

The prototype endoscopic probe developed in this study is based on a similar design, as described in our previous publications. ${ }^{8,9}$ It contains two stainless steel needles, the inner needle and the outer needle. Each of them houses one segment of gradient-index (GRIN) lens. The OCT laser beam is guided through a single-mode fiber into the inner needle. A glass ferrule is used to fix and center the fiber end inside the inner needle, and it is followed by the first GRIN lens. Both the front surface of the glass ferrule and the back surface of the lens have been angle-cut to $8^{\circ}$ to reduce reflection. The lens is $2.5-\mathrm{mm}$ long and collimates the incoming laser beam from the delivery fiber end. Its front end has been polished at a $22.5^{\circ}$ angle to initiate the first beam deflection.

The second GRIN lens, mounted in the outer needle, has a length of $5.6 \mathrm{~mm}$. It further deflects the beam and focuses it to a working distance ahead of the probe tip. The back end was also polished at a $22.5^{\circ}$ angle, and the front end was blunt and was sealed with epoxy to avoid fluid leakage.

The outer diameters of both lenses and the ferrule is $1.0 \mathrm{~mm}$. The inner/outer diameter (ID/OD) of the inner needle is $1.0 / 1.2 \mathrm{~mm}$, and that of the outer needle is $1.3 / 1.6 \mathrm{~mm}$. A metal sleeve was used to adapt the second lens into the outer needle, which has an ID/OD of $1.0 / 1.3 \mathrm{~mm}$. The overall length of the probe is $63.5 \mathrm{~mm}$. Optical grade epoxy was used to glue and fit the optical components inside the needles. The lenses were fabricated by GRINTECH GmbH (Jena, Germany), and the needle tubes were machined by Trinity Biomedical Inc.
(Menomonee Falls, WI). The schematic of the probe is illustrated in Figure 2 .

By rotating the inner and outer needles (thus the lenses) at the same angular speed but in opposite directions, the probe can steer the laser beam in a fan-shape pattern. Combined with the OCT axial scan, the probe can provide two-dimensional images representing physiological structures in the forward cone of the probe tip.

An actuation system was built to drive the needles, as partially pictured in Figure 3. The system uses a single motor and a set of bevel gears to mechanically ensure the rotation synchronization of the lenses. A feed-back electronic system was also implemented to maintain a constant rotation speed. The speed deviation was kept within $2.5 \%$ of the desired value. In the following imaging experiments, the system was configured to operate at 0.5 scans per second.

\section{Swept Source OCT System}

As shown in Figure 4, we built an OCT system based on a swept source laser (s3; Micron Optics, Atlanta, GA) for this study. The laser was centered at $1310 \mathrm{~nm}$, with a wavelength tuning range of approximately $100 \mathrm{~nm}$. Two optical circulators were used to assemble the interferometer for the OCT setup. The output power of the laser was $9.8 \mathrm{~mW}$, and the power delivered onto the sample was less than $1 \mathrm{~mW}$ because of the passive losses in the system. A 660-nm laser aiming beam with an average power approximately $10 \mu \mathrm{W}$ or less was combined with the OCT beam by a wavelength-division multiplexing coupler. It provided a visible indication of the scanning beam's position on the tissue. The A-scan rate was configured at $333 \mathrm{~Hz}$.

\section{Imaging the Collector Channel}

Human cadaver eye tissue was prepared for OCT imaging. The cornea and surrounding tissue were dissected from a whole globe, and the iris, lens, and supporting tissue were removed. The resultant shell was quartered, and the segments were dyed with methylene blue for a better visual contrast between the SC and the sclera. We removed TM from some tissue segments while maintaining TM for other segments.

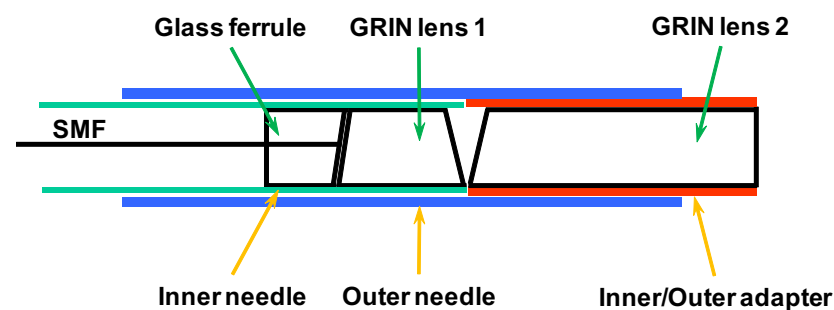

Figure 2. Schematic of the prototype endoscopic probe. SMF, singlemode fiber. 


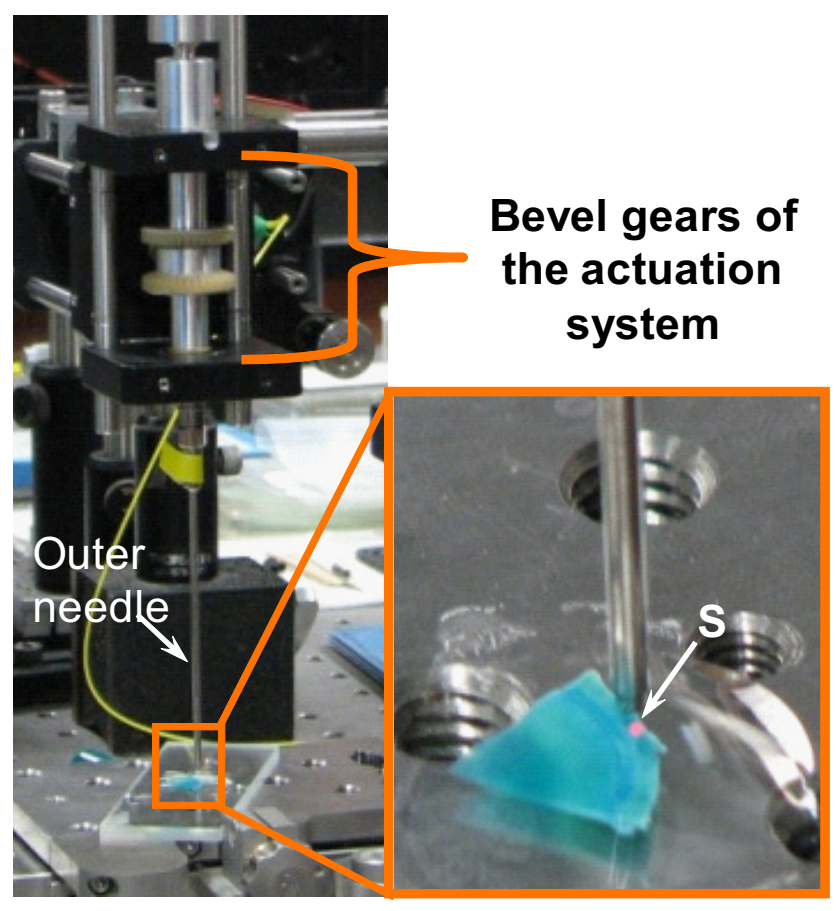

FIGURE 3. Side view of the endoscopic OCT probe and actuation system used in the CC imaging experiments. Close view of the probe tip and the human cadaver eye tissue underneath is enclosed. The sample has been dyed with methylene blue. The bevel gears of the actuation system synchronize the lens rotation mechanically. The outer needle is $63.5 \mathrm{~mm}$ long. $\mathrm{S}$, the red aiming beam spot onto the sample.

This formed two groups of samples. Each segment under test was placed in a microscope slide well with a few drops of basic saline solution to prevent desiccation. The microscope slide was secured on a mechanical stage. The OCT probe was positioned over the tissue and was adjusted to have a distance of approximately $1.5 \mathrm{~mm}$ from the tip to the sample, as shown in Figure 3. The stage could be translated to move the sample across the surface perpendicular to the probe's axis. OCT images were displayed on the system monitor.

First, we examined the tissue penetration of the $1310 \mathrm{~nm}$ OCT light on the samples with TM intact. To test the penetration over the entire sample, instead of rotating the two needles to generate a fan-shape scan, the stage was linearly translated so that the probe was traveling relatively perpendicular to SC by a longer range to cover the tissue sample while the laser beam was kept undeflected. Thus, the resultant OCT images in this step had a regular rectangular shape.

On the verification of the tissue penetration of $1310 \mathrm{~nm}$ light, we next proceeded to search and image CCs using the probe, where the needles were actuated to rotate. The stage was translated so that the

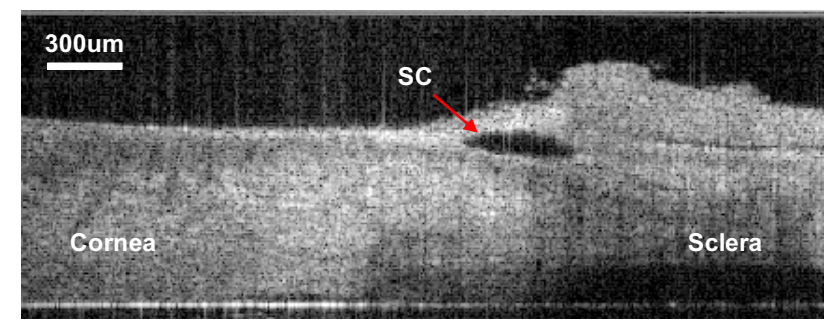

(a)

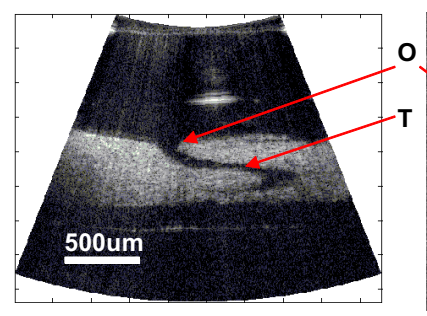

(b)

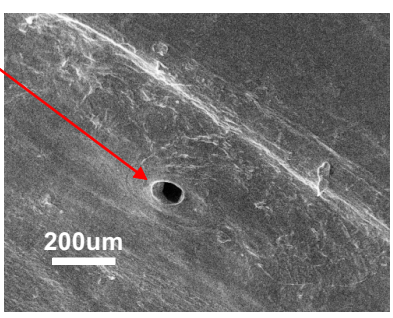

(c)
FIGURE 5. OCT and SEM images of human cadaver eye tissue segments. (a) OCT image of a tissue segment with TM intact, acquired by translating the stage. (b) OCT image of a tissue with TM removed, acquired by rotating the needles of the endoscopic probe. (c) SEM image of the same CC in (b). O, CC opening; T, CC path through sclera.

probe was moving relatively along the SC to search CCs while the beam was scanning the cross-section of the SC in a fan-shape pattern, as shown in Figure 1b. To provide corroboration of any CCs found by the probe, the experiments in this step were conducted on tissues with the TM removed so that image confirmation by scanning electron microscopy (SEM) could be performed later. If the TM had not been removed, it would have been very difficult to accurately map out the CCs found underneath across the entire sample and to image the appropriate parts of it by SEM. This would have resulted in a remote chance of correct correlation between OCT and SEM images. In addition, during SEM sample preparation, desiccation of the samples would have caused the TM tissue to obscure the openings of any CCs found during OCT examination.

Once we located the CCs from the OCT images, we recorded their locations and mapped them across the sample using the scanning visualization provided by the aiming beam. We then sent the sample for study by using SEM to image those spots where CC openings were located.

\section{Results}

A typical OCT image acquired by translating the stage in the penetration verification experiments is shown in Figure $5 \mathrm{a}$. It
Figure 4. The swept source OCT setup. SMF, single-mode fiber; PC, polarization controller; ADC, analog-to-digital converter; C, optical circulator.

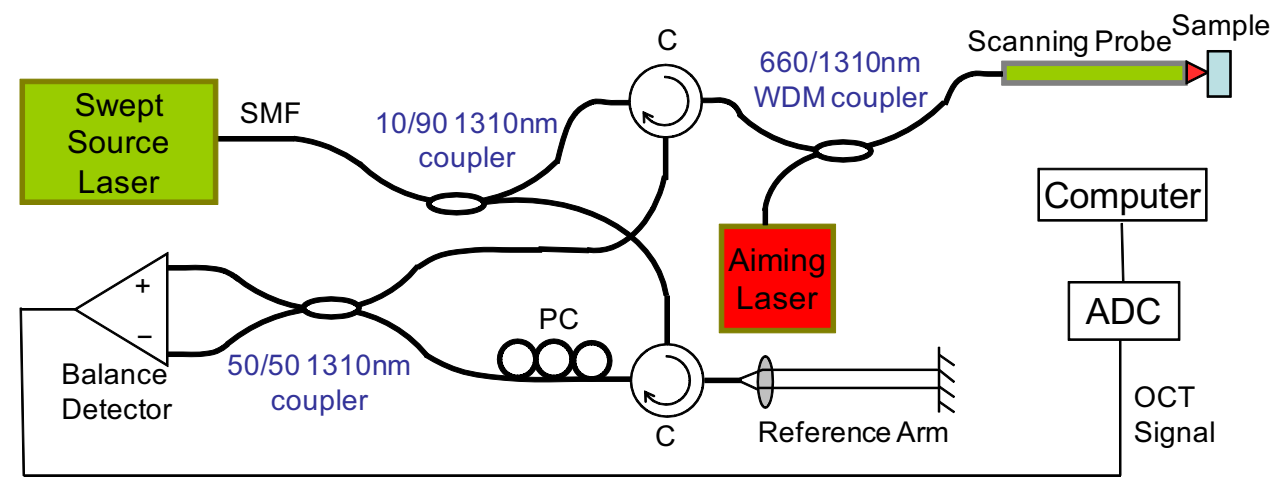


clearly depicts the shape of the cross-section of the SC. Tissues under the TM and on the other side of the SC wall have a clarity almost as good as that of the TM itself. There is no observable shadow effect from the TM. These images verify that the probe operating at $1310 \mathrm{~nm}$ can indeed clearly visualize tissues behind the TM for a considerable distance. Some of these images even reveal structures that could be CCs branching far away from their openings inside SC.

A typical OCT image acquired by rotating the needles of the endoscopic probe in the experiments searching CCs is shown in Figure 5b. From the OCT image, one can clearly see not only the CC opening exiting for the SC wall but also the shape of the channel winding into the sclera. Based on these images, we were able to quantitatively measure the dimensions of these physiological structures. The SEM image of the CC is shown in Figure $5 \mathrm{c}$ for comparison. The two methods agree with each other, indicating that the CC opening was approximately 120 $\mu \mathrm{m}$ wide.

The probe focuses the probing beam to a working distance of approximately $1.4 \mathrm{~mm}$ (in air) ahead of the tip. The focal spot was measured to range from 12 to $14 \mu \mathrm{m}$ in diameter. Both the working distance and the spot size have a weak dependence on the deflection angle. The maximum variation of the spot size was $16 \%$, and the variation in the working distance was $13 \%$.

A maximum scan range of approximately $40^{\circ}\left(20^{\circ}\right.$ for half angle) was achieved. The axial scan range was $3 \mathrm{~mm}$, which was determined by the sampling period in the wavenumber space. The signal-to-noise ratio of the entire system was measured to be grater than $95 \mathrm{~dB}$.

\section{Discussion}

As opposed to approximately $800 \mathrm{~nm}$, the typical wavelength range for ophthalmic OCT imaging used in previous studies (Kagemann L, et al. IOVS 2009;50:ARVO E-Abstract 813), ${ }^{4}$ a central wavelength of $1310 \mathrm{~nm}$ was selected for this study. Indeed, axial resolution is lower for longer central wavelength assuming the same wavelength scan range. The theoretical axial resolution of our system is $7.5 \mu \mathrm{m}$, whereas that of the previous studies (Kagemann L, et al. IOVS 2009;50:ARVO E-Abstract 813$)^{4}$ was $1.3 \mu \mathrm{m}$. However, as shown in Figure 5, one can easily identify and locate CCs from the images acquired by the probe. The $7.5-\mu \mathrm{m}$ axial resolution was sufficient because the dimensions of the structures were usually on the order of 100 microns. The fact that the previous systems (Kagemann L, et al. IOVS 2009;50:ARVO E-Abstract 813$)^{4}$ with higher axial resolution could not provide images of the same quality could have been due primarily to light scattering and absorption by intervening tissues. Although no blood was involved in this ex vivo study, one can expect an improved performance over shorter wavelengths for in vivo studies because shorter wavelengths are more greatly affected by optical scattering. ${ }^{6}$ Higher water absorption at $1310 \mathrm{~nm}$ should not impair image contrast as much as expected in external OCT systems because the distance from the probe tip to the tissue of interest is usually in the 0.5 - to $1-\mathrm{mm}$ range during the intended endoscopic imaging procedures.

This wavelength selection of $1310 \mathrm{~nm}$ has been verified in the initial penetration verification experiments. Although we did not happen to capture a CC during those experiments (which were not designed to search CCs after all), based on the images we obtained with TM intact, the clarity should have been comparable if a CC had been captured. In addition to the advantages of $1310 \mathrm{~nm}$ light, this occurred primarily because the beam has bypassed most of the intervening tissues; the only remaining tissue is a thin, flimsy layer of TM with a thickness usually around 10-20 microns that can be easily penetrated, as shown in Figure $5 \mathrm{a}$.

Although the rotation speed of the motor was well maintained as constant, the angular speed of the deflection was not constant because the deflection angle is not linearly related to the rotation angle. ${ }^{8}$ An accurate relationship between the two angles is critical for accurate image reconstruction. In this study, which was based on a simplified model, ${ }^{8}$ we developed a more accurate theoretical model to estimate the deflection angle. A numerical simulation (ZEMAX Development Corporation, Bellevue, WA) was also used to verify this calculation. Finally, we experimentally measured the relationship. These results are shown in Figure 6. We applied this result to the final image reconstruction to obtain the correct geometry of the structures.

In this endoscopic probe design, the actuation system is located far away from the probe tip, which enables easy miniaturization. The diameter of the probe was limited primarily by that of the GRIN lens. We had already achieved a narrower probe 9 encased in two needles of 23 and 21 gauge, respectively, for the inner and outer needles. Now GRIN lenses with diameters smaller than $400 \mu \mathrm{m}$ are commercially available. This can further reduce the probe size, making it small enough to be introduced through a clear corneal incision for OCT visualization of CC location before or while implanting a bypass shunt.

As mentioned in our previous publication, ${ }^{8}$ inherent in this technology is the capability to perform volumetric scanning by using different rotation modes. By driving the lenses at different angular speeds and switching their rotation directions, we can engineer many volumetric scanning patterns in addition to the planar fan-shape scan pattern. Those patterns might permit visualization of larger areas, leading to more rapid identification of tissue structures.

The frame rate of the OCT system ( $0.5 \mathrm{fps})$ was limited by the scan rate of the swept laser. To accumulate enough A scans (666 depth scans in our case) for each OCT image frame, the rotation speed of our probe had to be kept at a constant value of $15 \mathrm{rpm}$, which is much lower than what it can support. OCT engines with A-scan rates greater than $100 \mathrm{kHz}$ have been demonstrated, ${ }^{10}$ and OCT systems greater than $20 \mathrm{kHz}$ are now commercially available as well. These could significantly increase the frame rate of our endoscopic system.

$\theta$ VS $\xi$

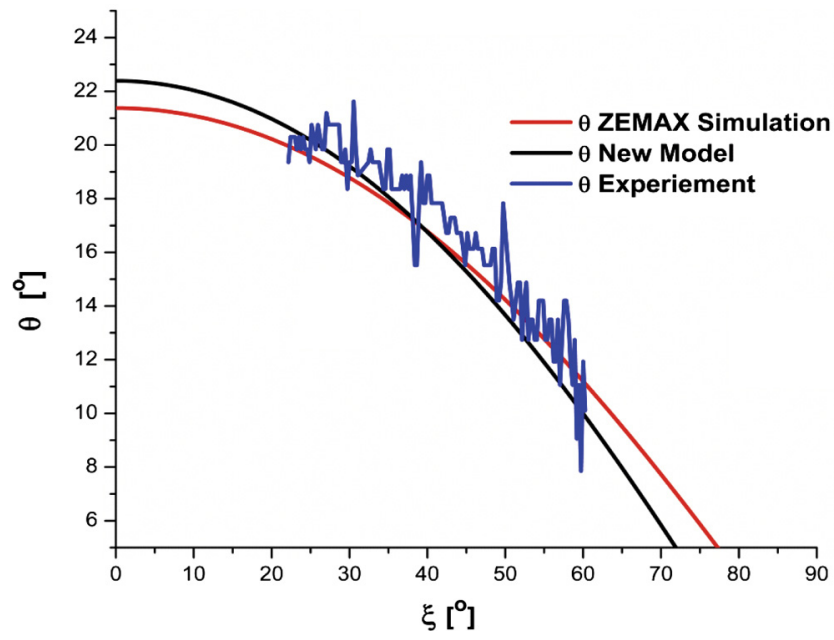

Figure 6. The relationship between deflection angle $\theta$ and rotation angle $\xi$. Black line: result of the new theoretical model; red line: result of the ZEMAX simulation; blue line: result of the experimental measurement. 


\section{Conclusion ANd Future Works}

This study demonstrated that our endoscopic probe has sufficient resolution to locate and image CCs exiting SC in ex vivo human cadaver eyes. It may potentially be adapted to visualize the anterior chamber intraoperatively to provide guidance for surgeries such as bypass stent implantation.

Motion artifact, a common problem in OCT images, results from the relative movement between tissue target and OCT instrument. During stent implantation, eyelids are usually immobilized by clamps. Because the probe is inserted through a corneal incision, the probe's position relative to the TM and SC are relatively constant. To further decrease this artifact, higher frame rates $(>24$ frames/s) must be achieved for real-time video operation by using OCT systems with faster A-scan rates. This upgrade, combined with improved eye immobilization procedures during surgery, will minimize the motion artifacts.

As mentioned, volumetric scan patterns can be designed to enable three-dimensional OCT imaging. Integration of this imaging device with other surgical tools may ultimately provide intraoperative assistance to surgical procedures that could benefit from real-time imaging guidance.

\section{Acknowledgments}

The authors thank Ying Wellsand of Thorlabs, Inc., whose connection made this research possible; Mike Roy of the Division of Chemistry and Chemical Engineering at the California Institute of Technology for help with the mechanical fabrication of the actuation system; and Kevin Hsu of Micron Optics, Inc., for the loan of the swept source laser.

\section{References}

1. Rosenquist R, Epstein D, Melamed S, Johnson M, Grant WM. Outflow resistance of enucleated human eyes at two different perfusion pressures and different extents of trabeculotomy. Curr Eye Res. 1989;8:1233.

2. Dvorak-Theobald G. Schlemm's canal: its anastomoses and anatomic relations. Trans Am Opbthalmol Soc. 1934;32:574.

3. Bahler CK, Smedley GT, Zhou J, Johnson DH. Trabecular bypass stents decrease intraocular pressure in cultured human anterior segments Am J Opbthalmol. 2004;138:988.

4. Kagemann L, Wollstein G, Ishikawa $H$, et al. Identification and assessment of Schlemm's canal by spectral-domain optical coherence tomography. Invest Ophthalmol Vis Sci. 2010;51:4054.

5. Huang D, Swanson EA, Lin CP, et al. Optical coherence tomography. Science. 1991;254:1178.

6. Povazay B, Bizheva K, Hermann B, et al. Enhanced visualization of choroidal vessels using ultrahigh resolution ophthalmic OCT at $1050 \mathrm{~nm}$. Opt Express. 2003;11:1980.

7. Ren J, Wu JG, McDowell EJ, Yang CH. Manual-scanning optical coherence tomography probe based on position tracking. Opt Lett. 2009;34:3400.

8. Wu JG, Conry M, Gu CH, Wang F, Yaqoob Z, Yang CH. Pairedangle-rotation scanning optical coherence tomography forwardimaging probe. Opt Lett. 2006;31:1265.

9. Han S, Sarunic MV, Wu J, Humayun M, Yang CH. Handheld forward-imaging needle endoscope for ophthalmic optical coherence tomography inspection. J Biomed Opt. 2008;13:020505.

10. Huber R, Adler DC, Srinivasan VJ, Fujimoto JG. Fourier domain mode locking at $1050 \mathrm{~nm}$ for ultra-high-speed optical coherence tomography of the human retina at 236,000 axial scans per second. Opt Lett. 2007;32:2049. 